\title{
Incidência de fratura do quadril em área urbana do Nordeste brasileiro
}

\author{
Hip fracture incidence in an urban area \\ in Northeast Brazil
}

Virgínia Angélica Lopes Silveira 1 Marta Maria das Chagas Medeiros 1 João Macedo Coelho-Filho 1

Rosa Salani Mota 2

Jamile Coelho Soares Noleto 1

Felipe Silveira da Costa 1

Francisco José Oliveira de Pontes 1

Juliana Barbosa Sobral 1

Raimundo Felipe Aguiar 1

Ângela Cristina Leal 1

Cristiano Magalhães Clemente 1

\section{Introdução}

1 Faculdade de Medicina, Universidade Federal do Ceará, Fortaleza, Brasil. 2 Centro de Ciências,

Universidade Federal do Ceará, Fortaleza, Brasil.

Correspondência M. M. C. Medeiros Departamento de Medicina Clínica, Faculdade de Medicina, Universidade Federal do Ceará. Rua Paula Ney 599, apto. 302, Fortaleza, CE 60140-200, Brasil. mmcmedeiros@hotmail.com

\section{Abstract}

Hip fractures involve high morbidity and mortality and extensive treatment costs and are thus considered an important public health issue. Hip fracture incidence varies greatly between countries and even between cities in the same region. This study aimed to determine hip fracture incidence in Fortaleza, Ceará State, Brazil. Hip fracture cases were identified prospectively in patients aged 45 and over attending private and public hospitals in Fortaleza from July 2001 to June 2002. The study identified 673 patients with hip fractures, 382 of whom from Fortaleza. Annual hip fracture incidence in Fortaleza for patients over 60 years was 21.7/10,000 inhabitants (13.0/10,000 for men and 27.7/10,000 for women). Hip fractures were more frequent among women and with advancing age. Hip fracture incidence in Fortaleza was very low compared to rates reported from other parts of the world.

Hip Fractures; Incidence; Risk Factors
O aumento crescente da expectativa de vida da população mundial tem contribuído para o aumento das doenças crônico-degenerativas na população idosa. A osteoporose assume especial atenção por se tratar de uma doença com alta prevalência entre os idosos, pelo risco maior de fraturas e pelo alto custo relacionado ao tratamento clínico-cirúrgico, tornando-a um grave problema de saúde pública. Dentre todos os sítios de fratura, as que ocorrem no quadril são as que apresentam maior impacto na morbidade e maior taxa de mortalidade, principalmente na população idosa. Estimativas apontam que no ano de 2050 ocorrerão aproximadamente 6,5 milhões de fratura de quadril em todo mundo 1 . A expectativa de vida dos pacientes que sofrem esse tipo de fratura é reduzida em 15,0 a $20,0 \%$, com um maior número de óbitos ocorrendo nos primeiros seis meses após o evento e as taxas de mortalidade por fraturas de quadril variam de 15,0 a 50,0\% no primeiro ano 2,3 . A incapacidade física total ou parcial após a fratura é um outro grande problema, chegando a 50,0\% dos pacientes confinados ao leito ou à cadeira de rodas e 25,0 a $35,0 \%$ daqueles que conseguem retornar ao domicílio precisam de cuidadores ou algum dispositivo para auxiliar a locomoção 4,5. Em países desenvolvidos como a Suécia, chega-se a gastar anualmente $40 \mathrm{mil}$ dólares por paciente com fratura de quadril 6 . 
A incidência de fratura de quadril varia muito nas diversas regiões do mundo, com taxas em mulheres e homens acima de sessenta anos, respectivamente, variando de 3 e 0,7 / 10 mil em Siena, Itália, até 122 e 50,1/10 mil em Oslo, Noruega. Na Europa observa-se gradiente de incidência de fraturas do norte para o sul, com taxas maiores na Noruega, Suécia, Dinamarca e taxas menores nas cidades em torno do Mediterrâneo 7,8,9. Na América do Sul, as taxas anuais de incidência de fratura de quadril em mulheres acima de cinqüenta anos variam de 9,4/10 mil na Venezuela a 44,9/10 mil no Chile 10,11,12. No Brasil, existem apenas dois estudos publicados realizados em cidades do interior das regiões Sudeste e Nordeste do país, ambos de natureza retrospectiva. A taxa de incidência de fraturas de quadril para pessoas acima de sessenta anos de idade na cidade de Marília, situada no Estado de São Paulo, Região Sudeste, em 1995 foi de 50,03/10 mil para o sexo feminino e de 18,73/10 mil para o sexo masculino 13 . Na cidade de Sobral, Ceará, entre os anos de 1996 a 2000, as taxas anuais para pessoas acima de sessenta anos foram de 20,7/10 mil para mulheres e 8,9/10 mil para homens 14 , taxas bem menores das registradas em Marília.

O objetivo do presente estudo foi determinar a taxa de incidência de fratura de quadril numa população residente em Fortaleza, uma capital do Nordeste brasileiro, utilizando um delineamento de estudo prospectivo e compará-la com a taxa de incidência no Sudeste brasileiro.

\section{Métodos}

Foram estudados todos pacientes com idade maior ou igual a 45 anos, de ambos sexos, que apresentaram diagnóstico de fratura do quadril no período de 1 de julho de 2001 a 30 de junho de 2002 e que procuraram atendimento em algum hospital (público ou privado) na cidade de Fortaleza, Ceará, Brasil. A escolha da população acima de 45 anos de idade se baseou no fato de que esta é a faixa etária em que as mulheres já se aproximam da menopausa, correndo, portanto, maior risco de desenvolver osteoporose e conseqüentemente, fraturas. Casos de fratura de fêmur de natureza neoplásica foram excluídos do estudo. O estudo foi prospectivo. Todos os hospitais públicos e privados do $\mathrm{Mu}$ nicípio de Fortaleza que atendiam pacientes com fratura do quadril foram selecionados para o estudo. A relação das vinte instituições que participariam do estudo foi obtida através de pesquisa junto à Secretaria de Saúde do Estado do Ceará, que forneceu a lista dos hospitais da rede pública e privada conveniados com o SUS e também junto ao Sindicato dos Hospitais do Ceará para seleção dos estabelecimentos com atendimento exclusivo da rede privada. Todos diretores dos vinte hospitais consentiram a realização do estudo. Funcionários da recepção e do centro cirúrgico de cada hospital foram identificados previamente e solicitados a notificar aos pesquisadores da chegada de todo caso de fratura de quadril admitido no hospital. Além do mais, visitas periódicas, aproximadamente a cada dois dias, eram realizadas sistematicamente pelos pesquisadores em cada hospital para que não ocorresse perda de nenhum caso. Após identificado o paciente, dados demográficos (sexo, idade) e procedência eram coletados. O pesquisador principal e sete estudantes do curso de medicina participaram do estudo de campo, ficando cada um responsável pela notificação dos casos de cada dois ou três hospitais. Semanalmente, o pesquisador principal escolhia aleatoriamente algumas instituições para validar dados registrados pelos estudantes. Somente pacientes procedentes de Fortaleza entraram para o cálculo da incidência. Os números referentes a população de Fortaleza foram fornecidos pelo Instituto Brasileiro de Geografia e Estatística (IBGE) de acordo com o último censo realizado em 2000.

\section{Resultados}

Durante o período do estudo, 673 indivíduos preencheram os critérios de inclusão do estudo, sendo que somente 382 eram provenientes da capital e o restante, de outras cidades do interior do Estado. Dos 382 pacientes de Fortaleza, 95 eram do sexo masculino $(24,9 \%)$ e 287 do sexo feminino $(75,1 \%)$, com uma proporção masculino/feminino de 1:3,02. A média de idade do grupo de pacientes estudado foi de 77,5 anos (desvio padrão - DP = 10,8; idade míni$\mathrm{ma}=49$ anos; idade máxima $=101$ anos).

A taxa de incidência de fratura nos pacientes acima de 45 anos foi de 9,35/10 mil habitantes, porém, quando consideramos apenas os pacientes acima de 60 anos, a incidência anual de fratura de quadril foi de 21,78/10 mil habitantes, sendo 13,00/10 mil para o sexo masculino e 27,50/10 mil para o sexo feminino.

Agrupando-se as fraturas, por faixas etárias e sexo, observa-se relevante aumento da incidência com a idade e no sexo feminino (Tabela $1 \mathrm{e}$ Figura 1). Apenas dois pacientes do sexo masculino fraturaram o quadril antes dos cinqüenta anos e todos os dois casos estiveram associados a trauma. 
Taxa de incidência de fratura do quadril, por sexo e faixa etária. Fortaleza, Ceará, Brasil, no período de julho de 2001 a julho de 2002.

\begin{tabular}{|c|c|c|c|c|c|c|}
\hline \multirow{2}{*}{$\begin{array}{l}\text { Faixa etária } \\
\text { (anos) }\end{array}$} & \multicolumn{2}{|c|}{ Total de casos de fratura } & \multicolumn{2}{|c|}{ População de Fortaleza } & \multicolumn{2}{|c|}{ Incidência* } \\
\hline & Homens & Mulheres & Homens & Mulheres & Homens & Mulheres \\
\hline $45-49$ & 2 & 0 & 45.889 & 57.316 & 0,43 & 0,00 \\
\hline $50-59$ & 11 & 20 & 63.654 & 81.212 & 1,73 & 2,46 \\
\hline $60-69$ & 14 & 35 & 36.277 & 52.128 & 3,85 & 6,71 \\
\hline 70-79 & 25 & 91 & 19.803 & 31.439 & 12,62 & 28,94 \\
\hline$>80$ & 43 & 141 & 7.018 & 13.566 & 61,27 & 103,93 \\
\hline Total & 95 & 287 & 172.641 & 235.661 & 5,50 & 12,17 \\
\hline
\end{tabular}

* Taxa de incidência por 10 mil habitantes.

Figura 1

Incidência de fratura do quadril por faixa etária e por sexo. Fortaleza, Ceará, Brasil,

entre julho de 2001 e junho de 2002.

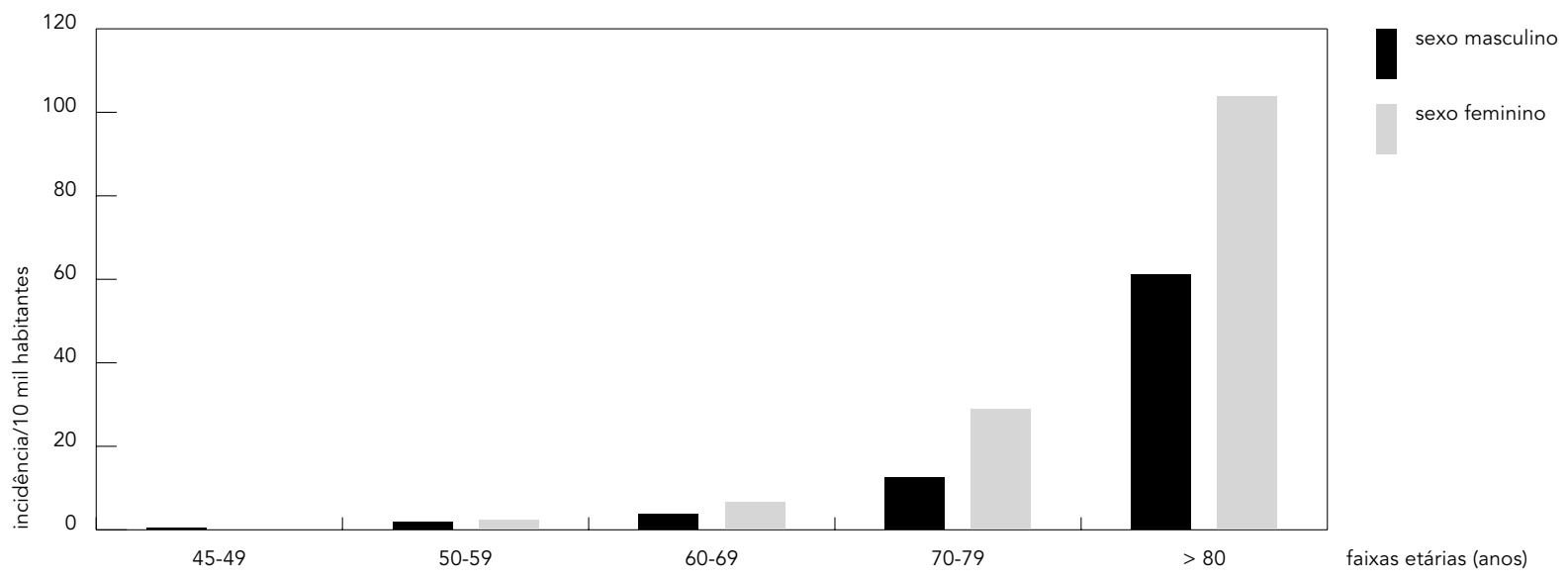

\section{Discussão}

A determinação das incidências de fraturas do quadril em vários países e mesmo em várias localidades dentro de um mesmo país tem mostrado grande variabilidade nas taxas, levandose a se suspeitar de vários fatores que explicassem tais diferenças, principalmente os genéticos, climáticos, étnico-culturais, antropométricos e geográficos. $\mathrm{O}$ conhecimento desses fatores em cada população poderia ajudar os gestores das políticas de saúde a tomarem medidas preventivas com o objetivo de se reduzir uma condição com grande impacto epidemiológico e econômico.

O Brasil é um país de grande extensão geográfica, com variações climáticas e de exposição solar, formado por populações de diferentes origens raciais com distintos estilos de vida e características antropométricas. Dois estudos brasileiros realizados em diferentes regiões revelaram dados muito interessantes, com uma incidência muito maior na cidade de Marília, São Paulo 13, Região Sudeste, quando comparada com a taxa na cidade de Sobral, Ceará 14 , Região Nordeste. O presente trabalho foi o pri- 
meiro do Brasil a medir a incidência de fratura de quadril numa capital brasileira de forma prospectiva, identificando todos pacientes no momento da internação hospitalar, minimizando, dessa forma, as perdas de contabilização dos pacientes. Sem dúvida, existe a possibilidade de alguns pacientes terem recebido assistência médica no domicílio e, portanto, não terem entrado para o cálculo da incidência. No entanto, pelo fato da fratura do quadril ser uma condição aguda, incapacitante, requerendo cirurgia na grande maioria das vezes e o estudo ter sido realizado numa cidade com vários hospitais públicos e privados preparados para o tratamento dessa condição, é improvável a interferência destas perdas no cálculo da incidência. Estudo realizado em Pequim, China, validou a estimativa de fratura do fêmur baseada em ocorrências registradas em hospitais, entrevistando de forma aleatória 2.113 mulheres acima de cinqüenta anos, constatando que cerca de 97,0\% delas com antecedentes de fratura de quadril tinham sido tratadas em unidade hospitalar 15.

Considerando apenas os pacientes acima de sessenta anos, com maior risco de fraturas associadas à osteoporose, a incidência anual de fratura de quadril na cidade de Fortaleza nos anos de 2001/2002 para o sexo masculino foi de $13,00 / 10$ mil habitantes e para o sexo feminino foi de 27,50/10 mil habitantes. Na cidade de Marília, em 1995, a taxa calculada para mesma faixa etária foi de 18,73/10 mil para o sexo masculino e de 50,03/10 mil para o sexo feminino 13 e na cidade de Sobral, nos anos de 1996/ 2000, a incidência anual no sexo masculino foi de 8,90/10 mil e no sexo feminino de 20,70/10 mil habitantes 14. Comparando as três taxas de incidência de populações brasileiras, observamos que parece existir um gradiente de incidência, com as menores taxas de fraturas de quadril em cidades do Nordeste brasileiro (Sobral, depois em Fortaleza) e a maior em cidade do Sudeste do Brasil. Esse gradiente possivelmente é influenciado pelas condições climáticas das duas regiões estudadas. O Estado do Ceará localiza-se um pouco abaixo da linha do Equador, numa posição nitidamente tropical e, portanto, recebe maior influência da luz solar e temperaturas mais altas. As cidades de Sobral e Fortaleza localizam-se, respectivamente, $3^{\circ} \mathrm{de}$ latitude sul e $40^{\circ}$ de longitude leste e entre $3^{\circ}$ $30^{\prime}$ e $4^{\circ} 30^{\prime}$ de latitude sul e $38^{\circ}$ de longitude leste, com temperaturas médias anuais de $26 \mathrm{e}$ $27^{\circ} \mathrm{C}$ e influência solar a maior parte do ano, propiciando menor confinamento dentro do domicílio pela ausência de períodos de inverno rigoroso e dessa forma, maior exposição à luz solar e maior ação da vitamina D no metabolismo ósseo. Já a Região Sudeste do país onde se encontra Marília, fica mais distante da linha do Equador, apresentando temperaturas mais baixas, com períodos de inverno e menor influência da luz solar. Além das diferenças climáticas entre as regiões, outros fatores que poderiam explicar as diferentes taxas encontradas são os genéticos e raciais. Enquanto o Nordeste brasileiro apresenta grande contingente de pessoas miscigenadas com influência genética dos índios, negros e também de portugueses, a região sudeste recebeu mais influência dos povos europeus durante sua colonização, principalmente portugueses e italianos, e também de japoneses. Além do mais, a população do nordeste brasileiro apresenta características antropométricas diferentes da Região Sudeste, sendo os nordestinos mais baixos do que os indivíduos da Região Sudeste. A participação destes fatores climáticos, genéticos e raciais, no entanto, são meramente especulativos, necessitando-se da realização de outros estudos para confirmar ou não estas impressões.

Como Sobral dista $204 \mathrm{~km}$ de Fortaleza e é uma cidade menos desenvolvida do que Fortaleza, com menos recursos de saúde, imaginamos que poderia ter existido migração de pacientes de uma cidade para outra, por ocasião da fratura do quadril, o que poderia explicar uma menor incidência em Sobral. Realmente, durante o período do nosso estudo, encontramos quatro pacientes atendidos em Fortaleza provenientes de Sobral, que não entraram no cálculo da incidência de fratura de quadril na cidade de Fortaleza. Quando, no entanto, utilizando os dados apresentados no estudo de Rocha et al. 14 , recalculamos a incidência incorporando esses quatro pacientes, não ocorreu diferença nos resultados, afastando, assim, um possível fator de migração influenciando as diferentes taxas de incidência entre as duas cidades.

No contexto mundial, a incidência de fratura do quadril também é bastante variável, influenciada por diversos fatores. Na Europa, observa-se gradiente de incidência de fraturas do norte para o sul, com taxas maiores na Suécia e Noruega quando comparadas às taxas nas cidades em torno do Mar Mediterrâneo. A incidência por 10 mil habitantes em pacientes acima de sessenta anos foi de 118 para o sexo feminino e de 44 para o sexo masculino em Oslo, Noruega entre 1996/1997, porém em países do sul da Europa como na França, a incidência foi de 44,4 para o sexo feminino e de 18 para o sexo masculino em 1987 8,9. Um estudo comparando peso e índice de massa corporal (IMC) dos indivíduos de Oslo com indivíduos de ou- 
tras regiões da Europa encontrou que os primeiros (tanto homens quanto mulheres) são mais altos e apresentam IMC menores do que os segundos 16. Os autores acham que isso poderia explicar, em parte, a grande incidência de fratura de quadril na população de Oslo. Nos Estados Unidos, a distribuição geográfica das fraturas do quadril é também variável, tendo sido demonstrada em trabalho realizado no período de 1984 a 1987, que a incidência anual em mulheres brancas acima de 65 anos por mil habitantes foi mais alta no Sul (maior que 9,47) quando comparada ao Norte do país (menor que 7,22 ), porém outro estudo realizado em pacientes brancos acima de 65 anos em diversas regiões dos Estados Unidos evidenciou que a região nordeste central apresentava maior taxa de incidência de fratura de quadril quando comparada às outras regiões 17,18. Mautalen \& Pimarino 11 publicaram em 1997 estudo realizado em Buenos Aires, Argentina, com taxas de incidência de 37,9/10 mil habitantes no sexo feminino e 10,1/10 mil no sexo masculino nos pacientes acima de cinqüenta anos e sugeriram que estas taxas poderiam ser representativas da América do Sul. Diante da incidência menor encontrada em Sobral e Fortaleza, acreditamos que a taxa encontrada na Argentina provavelmente não é representativa da América do Sul como um todo. Tais diferenças podem ter sofrido influência de diversos fatores, como a grande dimensão geográfica da América do Sul, as variações climáticas, as diferentes influências raciais da população e ainda os hábitos alimentares e de atividade física. A menor incidência de fratura do quadril descrita no mundo foi em Siena, Itália, com taxa em pacientes acima de sessenta anos de 3/10 mil habitantes para sexo feminino e 0,7/10 mil habitantes para sexo masculino, nos anos de 19751985 7. Kanis et al. 19 demonstraram que em Siena, o consumo de drogas que agem no metabolismo ósseo era alto, particularmente cálcio e calcitonina, tanto nas mulheres com fratura de quadril quanto nos controles quando comparado com outras cidades, sendo um dos fatores que poderia influenciar a baixa incidência de fratura do quadril nessa cidade.

Quando comparamos as taxas de incidência de fratura do quadril no mundo com as taxas do Brasil, concluímos que as encontradas em Fortaleza e Sobral, cidades do Nordeste brasileiro, estão bem abaixo dos índices descritos em cidades americanas e européias (com exceção de Siena, Itália). Esses baixos índices de fratura do quadril em cidades do Nordeste brasileiro contrastam com as condições sócio-econômicas mais baixas da população dessa região quando comparadas com as de outras cidades. A indisponibilidade da densitometria óssea no setor público de saúde do nosso Estado, retardando o diagnóstico e tratamento da osteoporose e o custo elevado das drogas utilizadas no tratamento da osteoporose poderiam contribuir para o aumento dos casos de fraturas relacionadas à doença. As baixas taxas de fratura de quadril no nosso Estado sugerem que fatores climáticos, genéticos, raciais e talvez antropométricos possam ter papel mais importante na osteoporose e, conseqüentemente, na fratura do quadril do que os fatores sócio-econômicos e culturais da população.

Quanto ao sexo, a fratura do quadril é mais freqüente nas mulheres nos diversos trabalhos realizados no mundo indicando que o sexo feminino é mais exposto aos fatores de risco, pela maior prevalência de osteoporose (menor pico de massa óssea e maior perda na pós-menopausa), maior susceptibilidade para quedas, maior expectativa de vida do que os homens, além das influências antropométricas e fatores genéticos relacionados ao sexo. No estudo atual a proporção homem/mulher nos pacientes acima de cinqüenta anos foi de 1:3,08, ligeiramente maior que a proporção da cidade de Siena $(1: 2,8) 7$ e um pouco menor que a encontrada nas cidades argentinas de La Plata e Buenos Aires $(1: 3,7)$ e no Chile $(1: 3,2) 11$. Portanto, poderíamos concluir que as proporções de homens e mulheres acima de cinqüenta anos que fraturaram o quadril são muito semelhantes entre as várias cidades estudadas. Outro fator importante na determinação das fraturas é a idade do indivíduo, pois a incidência da fratura do quadril aumenta com a idade, como demonstrado no presente estudo e em várias outras publicações anteriores. Nos pacientes acima de 80 anos, encontramos taxa de incidência aproximadamente dez vezes maior que naqueles com idade acima de 45 anos e quase quatro vezes maior que em pacientes do grupo etário imediatamente inferior (70-79 anos).

Em conclusão, a incidência de fratura de quadril na cidade de Fortaleza (Ceará, Nordeste do Brasil) é menor do que em cidade do Sudeste brasileiro e se encontra entre as menores taxas de incidência do mundo. Estudos semelhantes em cidades de outras regiões brasileiras são importantes para se confirmar ou não possível gradiente de incidência em nosso país, além de se esclarecer possíveis fatores protetores para fratura de quadril. 


\section{Resumo}

A fratura do quadril causa grande morbidade e mortalidade e envolve alto custo, sendo reconhecida como um problema de saúde pública. Sua incidência varia amplamente entre vários países e mesmo entre cidades de uma mesma região. O objetivo do estudo foi determinar a taxa de incidência de fratura de quadril na cidade de Fortaleza, Ceará, Brasil. Foram identificados prospectivamente todos casos de fratura de fêmur, em indivíduos acima de 45 anos, atendidos nos hospitais públicos e privados da cidade de Fortaleza no período de julho de 2001 a junho de 2002. Seiscentos e setenta e três pacientes fraturaram o fêmur, sendo 382 provenientes da cidade de Fortaleza. A incidência anual de fratura de quadril na cidade de Fortaleza em indivíduos acima de 60 anos foi de 21,7/10 mil habitantes (13/10 mil no sexo masculino e 27,7/10 mil no sexo feminino). As fraturas de quadril foram mais freqüentes no sexo feminino $e$ aumentaram progressivamente com a idade. A taxa de incidência do quadril na cidade de Fortaleza foi uma das mais baixas quando comparadas com estudos realizados em várias partes do mundo.

Fraturas do Quadril; Incidência; Fatores de Risco

\section{Referências}

1. Cooper C. Epidemiology of osteoporosis. Osteoporos Int 1999; 2 Suppl:2-8.

2. Fitzpatrick P, Kirke PN, Daly L, Vanrooij J, Dinn E, Birke $\mathrm{H}$, et al. Predictors of first hip fracture and mortality post fracture in older women. Ir J Med Sci 2001; 170:49-53.

3. Leibson CL, Tosteson AN, Gabriel SE, Ransom JE, Melton LJ. Mortality, disability, and nursing home use for persons with and without hip fracture: a population-based study. J Am Geriatr Soc 2002; 50:1644-50.

4. Cummings SR, Melton LJ. Epidemiology and outcomes of osteoporotic fractures. Lancet 2002; 359:1761-7.

5. Miller CW. Survival and ambulation following hip fracture. J Bone Joint Surg 1978; 60:930-4.

6. Zethraeus N, Stromberg L, Jonsson B, Suensson O, Ohlen G. The cost of a hip fracture. Estimates for 1,709 patients in Sweden. Acta Orthop Scand 1997; 68:13-7.

7. Agnusdei D, Camporeale A, Gerardi D, Rossi S, Bocchi L, Gennari C. Trends in the incidence of hip fracture in Siena, Italy, from 1980-1991. Bone 1993; 14:31-4.

8. Baudoin C, Fardellone P, Potard V, Sebert JL. Fractures of the proximal femur in Picardy, France in 1987. Osteoporos Int 1993; 3:43-9.

9. Lofthus CM, Osnes EK, Falch JA, Kaastad TS, Kristiansen IE, Nordsletten L, et al. Epidemiology of hip fractures in Oslo, Norway. Bone 2001; 29:413-8.

10. Bacon WE, Maggi S, Looker A, Harris T, Nair CR, Giaconi J, et al. International comparison of hip fracture rates in 1988-89. Osteoporos Int 1996; 6:69-75.

11. Mautalen C, Pimarino H. Epidemiology of osteoporosis in South America. Osteoporos Int 1997; 7(Suppl 3):S73-7.

\section{Colaboradores}

V. A. L. Silveira coletou e revisou dados da pesquisa, realizou tabulações, participou da edição e revisão da versão final do artigo. M. M. C. Medeiros revisou dados da pesquisa, redigiu a versão preliminar do trabalho, participou da edição e revisão da versão final do artigo. J. M. Coelho-Filho revisou dados da pesquisa, participou da edição e revisão da versão final do artigo. R. S. Mota realizou análises dos dados. J. C. S. Noleto, F. S. Costa, F. J. O. Pontes, J. B. Sobral, R. F Aguiar, A. C. Leal, C. M. Clemente coletaram dados da pesquisa

12. Pumarino H, Contreras L, Kirshbaum A. Tendencia de las tasas de fractura de cadera en 12 años en Chile. $i$ Aumento independiente de envejecimiento poblacional? Rev Med Chil 1997; 125:893-8.

13. Komatsu RS, Simões MFJ, Ramos LR, Szejnfeld VL. Incidencia de fraturas de fêmur proximal em Marília, São Paulo, Brasil, 1994 e 1995. Rev Bras Reumat 1999; 39:325-31.

14. Rocha FAC, Ribeiro AR. Low incidence of hip fractures in an equatorial area. Osteoporos Int 2003; 14:496-9.

15. Ling X, Almin L, Xile Z, Cummings SR. Very low rates of hip fracture in Beijing, People's Republic of China the Beijing Osteoporosis Project. Am J Epidemiol 1996; 144:901-7.

16. Meyer HE, Falch JA, O’Neill T, Tverdal A, Varlow J. Height and body mass index in Oslo, Norway, compared to other regions of Europe: do they explain differences in the incidence of hip fracture? European Vertebral Osteoporosis Study Group. Bone 1995; 17:347-50.

17. Bacon WE, Smith GS, Baker SP. Geographic variation in the occurrence of hip fractures among the elderly white US population. Am J Public Health 1989; 79:1556-8.

18. Jacobsen SJ, Goldberg J, Miles TP, Brody JA, Stiers W, Rimm AA. Regional variation in the incidence of hip fracture. US white women aged 65 years and older. JAMA 1990; 264:500-2.

19. Kanis JA, Johnell O, Gullberg B, Allander E, Dilsen G, Gennari C, et al. Evidence for efficacy of drugs affecting bone metabolism in preventing hip fracture. BMJ 1992; 305:1124-8.

Recebido em 20/Mai/2004

Versãofinal reapresentada em 19/Nov/2004 Aprovado em 07/Dez/2004 